\title{
Presynaptic protein synthesis required for NT-3-induced long-term synaptic modulation
}

\author{
H Shawn Je $\mathrm{e}^{1,2+}$, Yuanyuan $\mathrm{Ji}^{1,3+}$, Ying Wang ${ }^{4,5}$, Feng Yang ${ }^{1}$, Wei Wu ${ }^{4,5}$, Bai $\mathrm{Lu}^{1,3,4^{*}}$
}

\begin{abstract}
Background: Neurotrophins elicit both acute and long-term modulation of synaptic transmission and plasticity. Previously, we demonstrated that the long-term synaptic modulation requires the endocytosis of neurotrophinreceptor complex, the activation of PI3K and Akt, and mTOR mediated protein synthesis. However, it is unclear whether the long-term synaptic modulation by neurotrophins depends on protein synthesis in pre- or postsynaptic cells.

Results: Here we have developed an inducible protein translation blocker, in which the kinase domain of protein kinase $\mathrm{R}$ (PKR) is fused with bacterial gyrase B domain (GyrB-PKR), which could be dimerized upon treatment with a cell permeable drug, coumermycin. By genetically targeting GyrB-PKR to specific cell types, we show that NT-3 induced long-term synaptic modulation requires presynaptic, but not postsynaptic protein synthesis.
\end{abstract}

Conclusions: Our results provide mechanistic insights into the cell-specific requirement for protein synthesis in the long-term synaptic modulation by neurotrophins. The GyrB-PKR system may be useful tool to study protein synthesis in a cell-specific manner.

\section{Background}

Synaptic plasticity, or activity-dependent morphological and functional modification of synaptic connections, is the dominant underlying mechanism for brain function [1]. Recently, neurotrophins, a family of structurally and functionally related proteins, that include nerve growth factor (NGF), brain derived neurotrophic factor (BDNF), neurotrophin-3 (NT-3), and neurotrophin-4/5(NT-4/5), have emerged as major modulators involved in synaptic plasticity [2-4]. Similar to synaptic plasticity, synaptic effects of neurotrophins can be divided into two temporally distinct modes: the acute effect occurring within seconds or minutes upon a neurotrophin exposure, and the long-term effect taking hours and days to accomplish [5-7]. Previously, we identified that the acute and long-term effects of NT-3 are operated by distinct molecular and cellular mechanisms by using Хenopus cultured neuromuscular synapse $[6,8]$. Compared to acute effects, NT-3 mediated long-term synapse modulation

\footnotetext{
* Correspondence: bai.b.lu@gsk.com

+ Contributed equally

${ }^{1}$ Genes, Cognition and Psychosis Program (GCAP), National Institute of

Mental Health, NIH, Bethesda, MD 20892, U.S.A

Full list of author information is available at the end of the article
}

requires endocytosis of NT-3-TrkC (a cognate receptor for NT-3) complex, activation of Akt, a major downstream kinase of PI3K pathway, and mTOR dependent protein synthesis [6].

The requirement for protein synthesis assumes that NT-3 can trigger protein synthesis which can occur in presynaptic neurons or postsynaptic muscle cells [9]. Because conventional pharmacology cannot inhibit protein synthesis in a cell-type specific manner, we developed and utilized an inducible protein translation blocker that can be genetically targeted to specific cells to further investigate whether NT-3 induced long-term synaptic modulation requires either presynaptic or postsynaptic protein synthesis [10]. Our protein synthesis inhibitor system utilizes the double-stranded (ds) RNA-dependent protein kinase (PKR), which reversibly phosphorylates the $\alpha$ subunit of eukaryotic initiation factor- 2 (eIF2 $\alpha$ ) to control protein synthesis in eukaryotic cells [11]. The kinase activity of PKR is very low at rest, but is significantly induced upon binding of its dsRNA-binding domains to dsRNAs during viral infection, leading to dimerization, autophosphorylation, activation of the kinase, and eventual blockade of general mRNA translation [12]. To establish an inducible system, we utilized 
bacterial gyrase B domain, which could be dimerized upon treatment with a cell permeable drug, coumermycin [13]. By using this unique system that allows specific inhibition of general mRNA translation only on expressing cells, we show that NT-3 induced long-term synaptic modulation requires presynaptic, but not postsynaptic protein synthesis. Taken together, these results suggest general principles that govern long-term regulation of synapses by neurotrophins.

\section{Results}

\section{GyrB-PKR, an inducible molecular system to block protein synthesis}

Previously, we found that the rapamycin (200 nM), a specific inhibitor for mTOR, blocked NT-3 induced longterm synapse modulation [6]. Pharmacological inhibitors may elicit side effects in addition to its inhibition of protein synthesis $[14,15]$. It is also unclear whether rapamycin acts pre- or postsynaptically. Here we attempted to develop a genetic approach to examine the importance of protein synthesis in NT-3-induced synaptic modulation. The dimerization of PKR kinase domain has been shown to be both necessary and sufficient to activate its kinase function [13], which could suppress protein synthesis by phosphorylating eIF $2 \alpha$, leading to the dissociation of eIF2-tRNA-40 S complex [11]. We replaced dsRNAbinding domain of PKR with E. coli protein gyrase B, which could be dimerized upon exposure to the cellpermeable ligand coumermycin [16]. This fusion protein GyrB-PKR should therefore in theory confer inducible and reversible inhibition of protein synthesis upon treatment with coumermycin (Figure 1A).

To determine whether coumermycin truly induced dimerization and activation of GyrB-PKR, we expressed GyrB-PKR in developing Xenopus embryos by blastomere injection techniques [17]. Western blot analysis was used to monitor the expression of GyrB-PKR and phosphorylation of eIF2 $\alpha$, a direct downstream target of PKR, upon treatment with coumermycin at various concentrations and durations. Addition of $0.1 \mu \mathrm{M}$ coumermycin caused eIF2 $\alpha$ phosphorylation (Figure 1B). The half-maximum response $\left(\mathrm{EC}_{50}\right)$ value for coumermycininduced eIF $2 \alpha$ phosphorylation was $1 \mu \mathrm{M}$, which was measured 8 hours after drug treatment (Figure 1C). Coumermycin treatment led to a robust eIF $2 \alpha$ phosphorylation as early as $5 \mathrm{~min}$, which lasted more than 10 hours (Figure 1D and 1F). Moreover, when coumermycin was removed 2 hours after its application, the eIF $2 \alpha$ phosphorylation began to decline at 4-hour and reached baseline levels at 10-hour (Figure $1 \mathrm{E}$ and $1 \mathrm{~F}$ ). Taken together, these experiments indicate that the expression of GyrB-PKR results in inducible and reversible phosphorylation of eIF $2 \alpha$ upon coumermycin treatment.
Next, we investigated whether the dimerization and subsequent activation of PKR inhibits new protein synthesis. Due to limited number of cells available in these nerve-muscle co-cultures, it was not feasible to directly measure protein synthesis using conventional approaches, such as ${ }^{3} \mathrm{H}$-leucine incorporation. Thus, we utilized a destabilized green fluorescence protein (pd1EGFP, half live $=1$ hour) whose fluorescence fades if protein synthesis is blocked. In pd1-EGFP, the residues 422-461 of mouse ornithine decarboxylase (MODC) were fused to the $C$ terminus of EGFP to enable a rapid protein degradation and turnover [18]. Therefore, by measuring GFP fluorescence change, we could monitor steady-state levels of GFP proteins, which should correlate with the degree of general protein synthesis. When pd1-EGFP was expressed in spinal neurons by embryo injection (Figure 2A), treatment of the cultures with the general protein synthesis inhibitor rapamycin (200 nM) or cyclohexamide $(60 \mu \mathrm{M})$ for 1 hour greatly reduced fluorescence intensity as a consequence of the inhibition of new EGFP synthesis, which indicated the feasibility of monitoring protein synthesis using this assay (data not shown) [19].

To determine whether coumermycin treatment inhibits protein synthesis in cultured spinal neurons, we expressed pd1-EGFP with or without Gyr-PKR in Xenopus spinal neurons and monitored the changes in fluorescent intensity upon coumermycin treatment. Indeed, coumermycin treatment reduced the GFP fluorescent intensity by $45 \%$ in spinal neurons only when pd1-EGFP co-expressed with GyrB-PKR (Figure 2B). Taken together, these results demonstrate that coumermycin induced dimerization of PKR effectively phosphorylates eIF2 $\alpha$ and subsequently blocks new protein synthesis.

\section{Presynaptic protein synthesis in NT-3-induced synaptic modulation}

At the Xenopus neuromuscular synapses, application of exogenous NT-3 at a high concentration $(50 \mathrm{ng} / \mathrm{ml})$ induces a rapid potentiation of synaptic transmission within 5 min [20,21], whereas long-term treatment with a lower concentration of NT-3 (5 ng/ml; 2 days) facilitates physiological and morphological maturation of the synapses $[6,8,17]$. We recorded spontaneous synaptic currents (SSCs) in 1-d old nerve-muscle co-culture using whole-cell voltage clamp recording techniques. As reported, acute application of NT-3 elicited a marked increase in transmitter release in neurons (Figure 3A, bottom). The same treatment in the presence of coumermycin didn't affect NT-3 mediated acute effect, indicating that coumermycin itself did not affect synaptic transmission (Figure 3C, columns 3\&4).

The embryo injection technique allows selective expression of GyrB-PKR in either presynaptic motor 


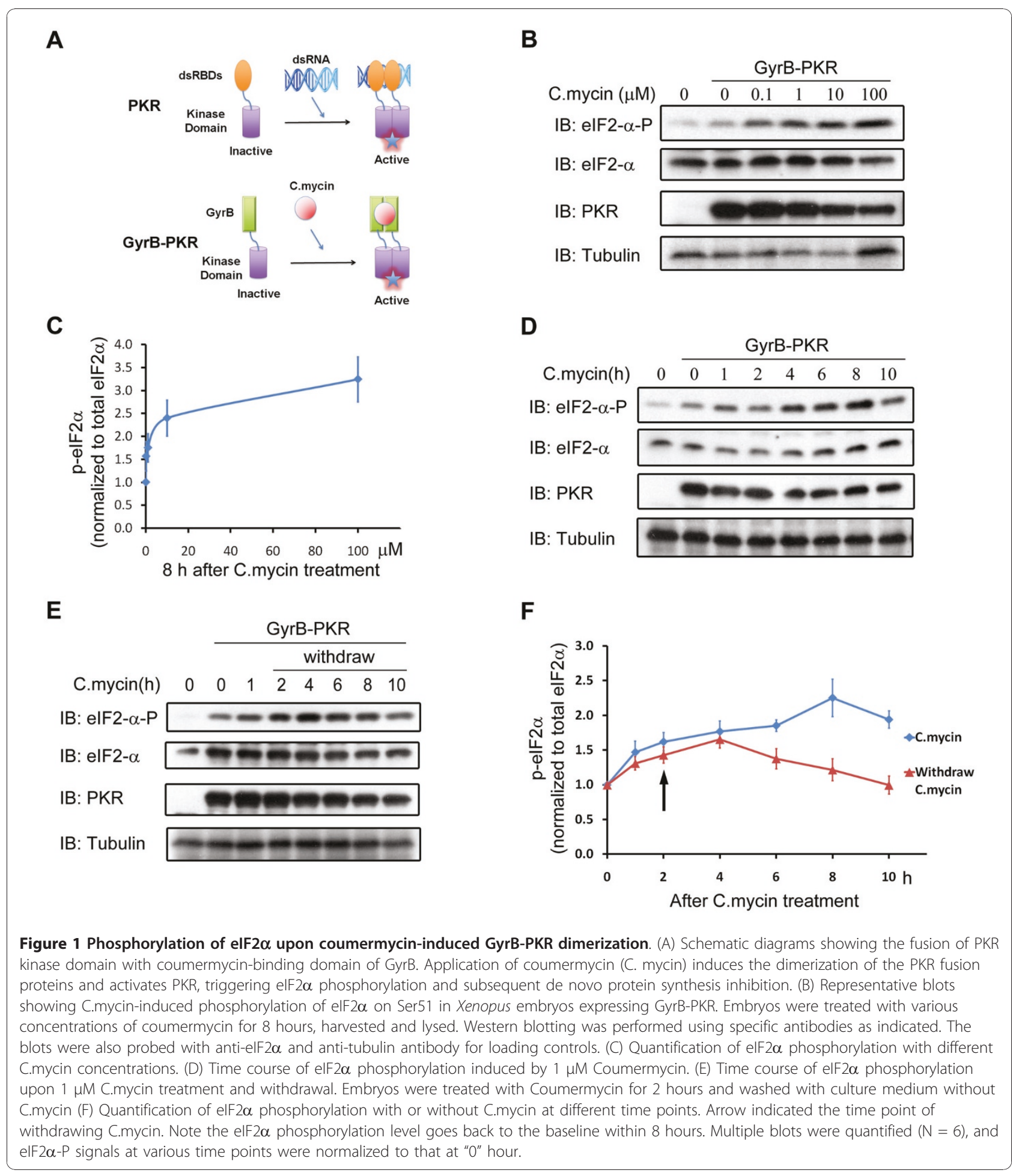

neurons or postsynaptic myocytes, as indicated by coexpressed GFP fluorescence, at neuromuscular synapses in the nerve-muscle co-culture (Figure 3B). Using this system, we tested whether activation of GyrB-PKR either presynaptically or postsynaptically alters the NT-3 effect. When GyrB-PKR was expressed in the postsynaptic muscle cells, application of NT-3 in the presence of coumermycin had no effect on the acute synaptic potentiation induced by NT-3 (Figure 3C, columns 5-8). Similarly, the expression of GyrB-PKR in presynaptic motor neurons also failed to alter the NT-3 effect in coumermycin treated cultures (Figure 3C, columns 9-12). These 


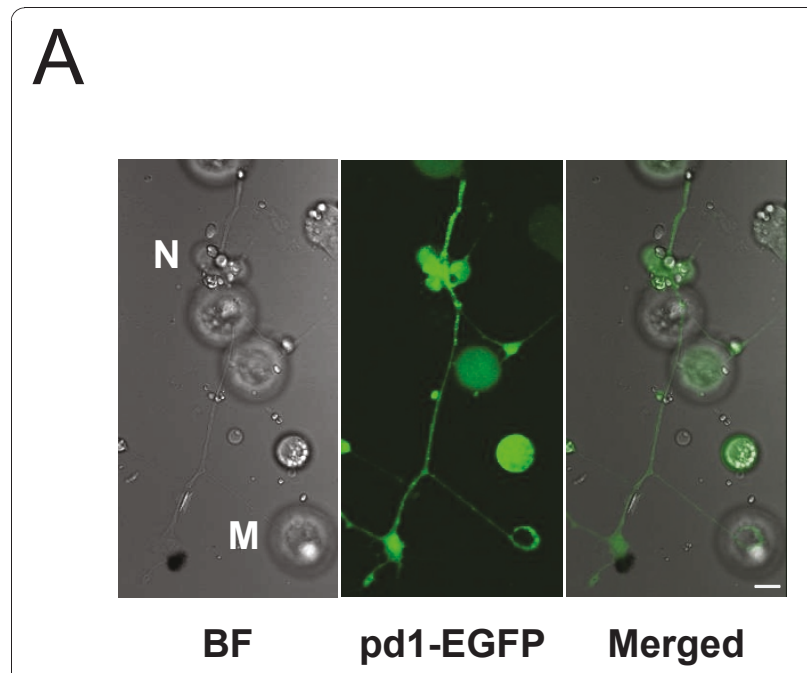

B

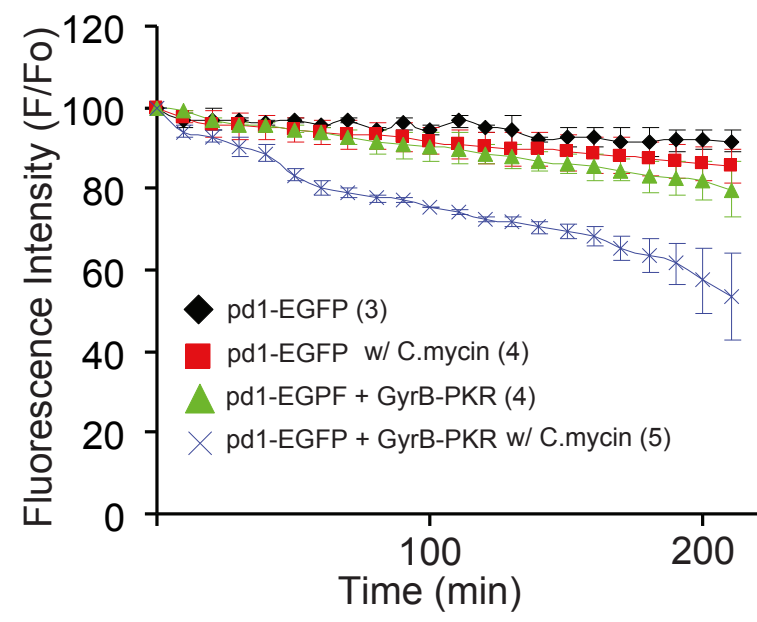

Figure 2 Inhibition of protein translation in spinal neurons by C.mycin -induced GyrB-PKR dimerization. (A) Expression of the pd1-EGFP in Xenopus spinal neurons. Bright field, fluorescent, and merged images (left, middle, right, respectively) show a spinal neuron and muscle cells expressing pd1-EGFP. N: neuron; M: muscle cell. Bar, $10 \mu \mathrm{m}$. (B) Quantification of fluorescence change on neurons. Pd1-EGFP was expressed either alone or together with GyrB-PKR in Xenopus spinal neurons, using embryo injection techniques. C.mycin was applied to the culture dish at time " 0 ", and pd1-EGFP fluorescence was monitored over time. The number of experiments performed was indicated in the legend.

results together suggest that the acute synaptic potentiation by NT-3 does not require protein synthesis.

Next, to determine whether pre- or post-synaptic protein synthesis is necessary for NT-3 mediated long-term synaptic modulation, we expressed GyrB-PKR in either spinal neurons or myocytes using the same embryo injection techniques described above. Cultures were incubated with NT-3 $(5 \mathrm{ng} / \mathrm{ml})$ for 2 days with or

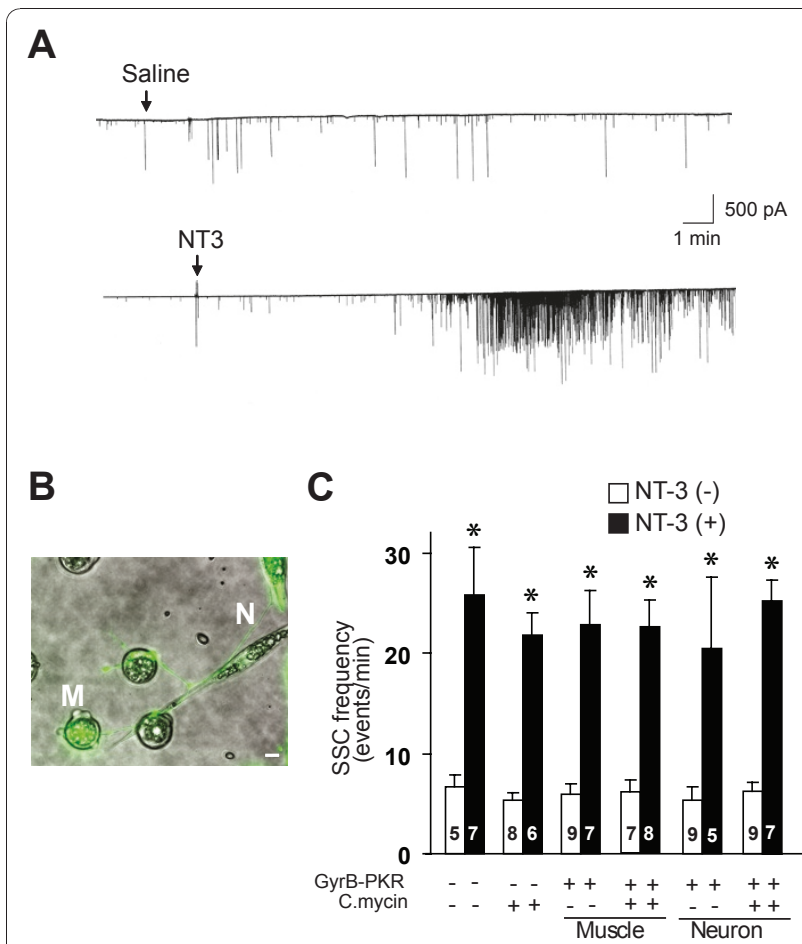

Figure 3 Normal acute synaptic potentiation by NT-3 in GyrBPKR activated neuromuscular synapses. (A) Sample recordings showing acute synaptic potentiation by NT-3. NT-3 $(50 \mathrm{ng} / \mathrm{ml})$ was applied directly to 1-day-old nerve-muscle co-culture. The frequency of spontaneous synaptic currents (SSCS) was used to monitor changes in synaptic efficacy. (B) A sample image showing neuromuscular synapses in which muscle cells are innervated by a GFP expressing spinal neuron. N: neuron axon (neuron body is out of this image); M: muscle cell. Bar, $10 \mu \mathrm{m}$. (C) Summary of acute effect of NT-3 on synapses without GyrB-PKR expression, and synapses with presynaptic or postsynaptic expression of GyrB-PKR. Each data point represents SSCs frequency (averaged from $30 \mathrm{~min}$ of recording) from a single synapse before C.mycin and after NT-3 application. Note that the application of coumermycin did not affect the baseline of recording. The number associated with each column represents the number of cells analyzed. Data are presented as the mean \pm the SEM.

without coumermycin as indicated (Figure 4A). At naive synapses, coumermycin treatment did not affect basal synaptic transmission nor prevent the long-term potentiating effect of NT-3 (Figure 4B, columns 3\&4). Expression of GyrB-PKR in either presynaptic spinal neurons (Figure 4B, columns $5 \& 6$ ) or postsynaptic muscle cells (Figure 4B, columns 9\&10) without coumermycin treatment did not alter the long-term effect of NT-3. Intriguingly, coumermycin treatment completely blocked the long-term effect of NT-3 in synapses made by spinal neurons expressing GyrB-PKR (Figure 4B, columns $11 \& 12)$. However, the same treatment was ineffective if GyrB-PKR was expressed in postsynaptic myocytes (Figure 4B, columns 7\&8). Taken together, these results suggest that protein synthesis in the presynaptic spinal 


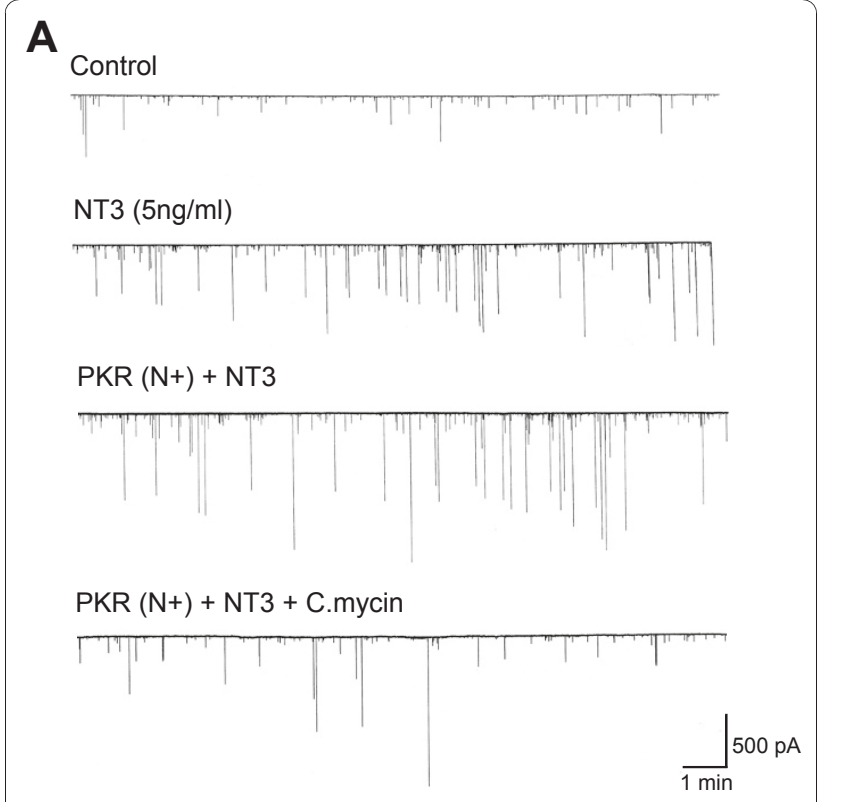

B

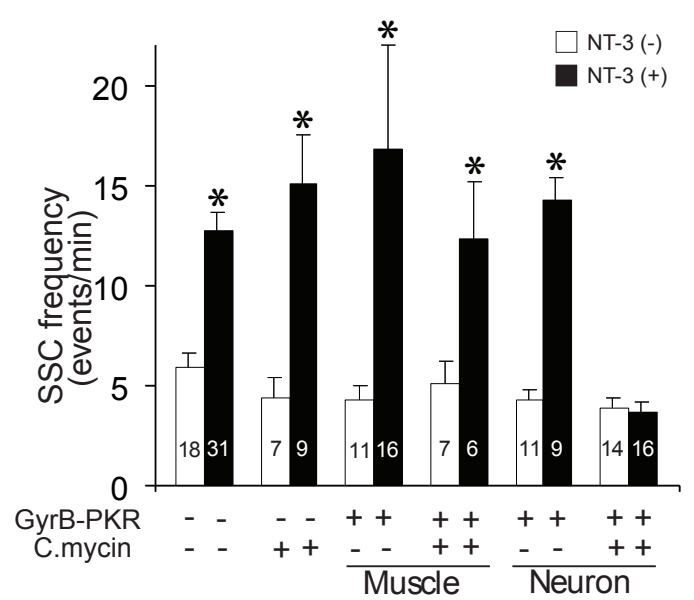

Figure 4 Blockade of the long-term synaptic effect of NT-3 by C.mycin -induced PKR activation. (A) Sample recordings showing long-term synaptic potentiation by NT-3. The Xenopus nerve-muscle co-cultures were grown in the presence or absence of NT-3 (5 ng/ $\mathrm{ml}$ ) for 2 days. Expression of GyrB-PKR alone did not prevent NT-3mediated long-term synaptic potentiation. However, application of C.mycin to synapses expressing GyrB-PKR presynaptically completely prevented long-term synaptic potentiation by NT-3. (B) Summary of long-term effect on NT-3 on synapses without GyrB-PKR expression, and synapses with pre- or post-syanptic expression of GyrB-PKR. Each data point represents SSCs frequency (averaged from $30 \mathrm{~min}$ of recording) form a single synapse with or without coumermycin treatment. Note that application of C.mycin alone could not attenuate the increase in SSC frequency by NT-3 chronic treatment. Application of C.mycin to synapses expression GyrB-PKR presynaptically, but not postsynaptically, completely prevented longterm synaptic effect of NT-3. The number associated with each column represents the number of cells analyzed. Data are presented as the mean \pm the SEM. neurons but not postsynaptic muscle cells is critical for NT-3 mediated long-term synaptic modulation at neuromuscular synapses.

\section{Discussion}

Targeting protein synthesis inhibition to specific cells

We have previously described an inducible PKR system that is based on dimerization of FKPB-PKR induced by the synthetic ligand AP20187 [10,22]. Here we report a similar system based on GyrB-PKR induced by coumermycin. Both systems have a major advantage over the conventional pharmacological inhibition of protein synthesis: genetically targeting to a specific cell population. This is particularly valuable in heterogeneous system in which cell-cell interaction is prominent, such as pre- and postsynaptic interactions in the nervous system. The GyrB-PKR system is attractive in several ways. First, coumermycin is an antibiotic that is not toxic to vertebrate cells. In our hands, incubation with coumermycin at $1 \mu \mathrm{M}$ for two days showed no obvious adversary effect to the nerve-muscle cultures (unpublished observations). Second, in the GyrB-PKR fusion construct, the dsRBD is removed and replaced it with GyrB, a bacterial protein that dimerizes upon binding to coumermycin. This modification prevents non-specific activation of PKR by other agents. Third, the only clearly verified substrate of PKR is the eukaryotic translation initiation factor eIF2 $\alpha$ [13]. Phosphorylation of Ser51 on eIF2 $\alpha$ converts it from a substrate to a competitive inhibitor of the guanine-nucleotide exchange factor eIF2B, blocking general mRNA translation. Activation of PKR therefore represents a specific inhibition of protein synthesis with relatively few side effects. Finally, using embryo injection techniques, we show that the GyrBPKR system is very useful in selective inhibition of protein synthesis in pre- or postsynaptic cells. Taken together, the GyrB-PKR system offers an alternative way to inducibly and reversibly block protein synthesis in the targeted cells, allowing applications in situations when AP20187 could not be used.

\section{Pre- and postsynaptic protein synthesis in synaptic modulation}

Compelling evidence suggests that one of the fundamental differences between acute and long-term synaptic modulation by neurotrophins is the requirement for protein synthesis. Acute potentiation of synaptic transmission by NT-3 is completely insensitive to translation inhibitors such as anisomycin and cycloheximide [23]. In contrast, the long-term form of NT-3 mediated synaptic modulation, including both structural (synaptic varicosity) and functional (synaptic currents) changes, requires protein synthesis. Bath application of rapamycin, a 
protein synthesis blocker by targeting mTOR, completely reversed the morphological and physiological changes induced by long-term exposure to NT-3 [6]. The major contribution of the present study is to demonstrate that at the developing neuromuscular synapses, it is the protein synthesis in the presynaptic motor neurons, but not in postsynaptic muscle cells, that mediates NT-3 induced long-term synaptic modulation. We show that coumermycin can effectively block the NT-3 effects only when the GyrB-PKR is selectively expressed in the pre- but not in postsynaptic cells. This finding indicates that NT-3 stimulates protein synthesis in presynaptic neurons, supplying the necessary proteins to enhance synaptic functions. It remains to be investigated whether the increase in protein synthesis occurs at the motor neuron soma, or in the motor axons or terminals. It should be note that protein synthesis in the presynaptic axon was reported to be involved in activity-dependent synaptic plasticity in sensory motor synapse in Aplysia and mature crayfish neuromuscular junctions [24,25].

Similar to neurotrophin-induced synaptic potentiation at the neuromuscular synapses, late-phase long-term potentiation (L-LTP) at the hippocampal CA1 synapses also depends on protein synthesis $[5,26]$. By injecting FKBP-PKR-expressing virus into CA1, but not CA3, of hippocampus in vivo, our previous study demonstrated that postsynaptic, but not presynaptic, inhibition of protein synthesis blocks L-LTP [10]. These results suggest that at the CA1 synapses in the hippocampus, protein synthesis in the postsynaptic CA1 neurons, rather than presynaptic CA3 neurons, is critical in maintaining L-LTP [10]. Thus, for long-term synaptic modulation, there is no set rule for the requirement of protein synthesis in pre- or postsynaptic site.

\section{Conclusion}

We developed an inducible protein synthesis blocker that can be genetically targeted to specific types of cells. By using this novel molecular tool, we have identified that presynaptic protein synthesis is crucial for NT-3mediated long-term synaptic modulation in Xenopus neuromuscular synapses. Our findings elucidate mechanistic insights into the cell-specific requirement for protein synthesis in the long-term synaptic modulation by neurotrophins.

\section{Methods}

DNA constructs, Xenopus embryo injection, nerve-muscle co-culture and whole-cell patch clamp recording

GyrB-PKR construct, which contains a bacterial gene GyrB fused with the kinase domain of PKR (GyrB-PKR, Figure 1A), was described previously [13]. Capped GyrB-PKR mRNAs were synthesized using mMessage machine (Ambion), mixed with GFP mRNA (1 mg/ml) in a 1:1 ratio, and injected into one blastomere at the 2- or 4-cell stage embryos using the Picospitzer pressure ejector as described [6].

Nerve-muscle cultures were prepared one day after injection [6]. Briefly, neural tubes and associated myotomal tissues of Xenopus embryos at stage 20 were dissociated in $\mathrm{Ca}^{2+}-\mathrm{Mg}^{2+}$-free medium $(58.2 \mathrm{mM} \mathrm{NaCl}$, $0.7 \mathrm{mM} \mathrm{KCl}$, and $0.3 \mathrm{mM}$ EDTA, $\mathrm{pH} \mathrm{7.4)} \mathrm{for} \mathrm{15-20}$ min. Cells were plated on clean glass coverslips, and grown in the presence or absence of NT-3 $(5 \mathrm{ng} / \mathrm{ml}$, gift from Regeneron Phamaceuticals) for 2 days at room temperature. Coumermycin, which induces GyrB-PKR dimerization, was added 1 hour before NT-3 treatment. The culture medium consisted (vol/vol) of $50 \% \mathrm{~L}-15$ medium, $1 \%$ fetal calf serum and 49\% Ringer's solution (117.6 mM NaCl, $2 \mathrm{mM} \mathrm{CaCl}, 2.5 \mathrm{mM} \mathrm{KCl}, 10 \mathrm{mM}$ HEPES, pH 7.6).

Synaptic currents were recorded from innervated muscle cells in 1 or 2-day old cultures by the whole-cell patch clamp recording in culture medium at room temperature [8]. The internal pipette solution contained $150 \mathrm{mM} \mathrm{KCl}, 1 \mathrm{mM} \mathrm{NaCl}, 1 \mathrm{mM} \mathrm{MgCl} 2$ and $10 \mathrm{mM}$ HEPES buffer ( $\mathrm{pH} 7.2$ ). The membrane potentials of the muscle cells recorded were generally in the range of -55 to $-75 \mathrm{mV}$ and were voltage clamped at $-70 \mathrm{mV}$. All data were collected by an Axonpatch 200B patch clamp amplifier (Molecular Devices), with a current signal filter set at $3 \mathrm{kHz}$. The frequency of spontaneous synaptic currents (SSCs) was defined as the number of SSC events per minutes. The frequency and amplitude of SSCs were analyzed using Clampfit software (Molecular Devices). Pipette and membrane capacitance and serial resistance were compensated.

\section{Western blot analysis}

Western blotting was performed as described [8]. Xenopus embryos at stage 20-22 were quickly homogenized in the extraction buffer $(100 \mathrm{mM} \mathrm{NaCl}, 50 \mathrm{mM}$ Tris$\mathrm{HCl}, \mathrm{pH} 7.5$, 1\% NP-40, $2 \mathrm{mM}$ PMSF, $1 \mathrm{mg} / \mathrm{ml}$ aprotinin, $1 \mathrm{mg} / \mathrm{ml}$ leupeptin, $1 \mathrm{mg} / \mathrm{ml}$ pepstatin $\mathrm{A}, 2 \mathrm{M}$ $\mathrm{Na}_{3} \mathrm{VO}_{4}$ ) and subsequently sonicated. The insoluble pellet after high-speed centrifugation was discarded and the resulting supernatants were transferred to fresh tubes containing $300 \mathrm{ml}$ freon (1,1,2-trichlorotrifluoroethane) (Sigma), vortexed for $1 \mathrm{~min}$, incubated on ice for $5 \mathrm{~min}$ utes, and subsequently centrifuged to remove yolk protein. Next, protein concentrations were determined using the Bradford protein assay kit (BioRad). Proteins were separated by SDS-polyacrylamide electrophoresis, and blotted onto Immobilon-P membrane (Millipore) with Semi-dry gel transfer apparatus (BioRad). The blots were incubated overnight at $4^{\circ} \mathrm{C}$ with primary antibodies including anti-PKR (1:1000) (Cell Signaling Technology), anti-phosphorylated form of eIF $2 \alpha$ (1:1000) (Assay 
designs), anti-tubulin (Covance), and anti-eIF $2 \alpha$ (Cell Signaling). Next, blots were incubated with secondary antibodies conjugated with HRP (Jackson Immunoresearch) and signals were detected by chemiluminescence kit (GE healthcare).

\section{Protein synthesis inhibition assay}

Destabilized EGFP vector (pd1EGFP-N1 from clontech) was used to monitor new protein synthesis in Xenopus spinal neurons. In the pd1EGFP, residues 422-461 of mouse ornithine decarboxylase (MODC) were fused to the $\mathrm{C}$ terminus of EGFP and this region of MODC contains a PEST amino acid sequence that targets the protein for degradation, resulting in rapid protein turnover. This PEST amino acid sequence of MODC is highly conserved in Xenopus, mice and human. It is correlated with most short-lived proteins [27]. pd1EGFP has a half-life of approximately one hour, as measured by fluorescence intensity of cells treated with the protein synthesis inhibitor cycloheximide [18]. pd1EGFP and/or GyrB-PKR were expressed in Xenopus spinal neurons by embryo injection. Images were collected with $40 \times$ objective lens (NA 1.0) on a fluorescence microscopy. Fluorescence intensity from a small region of interest (ROI, 6 pixels by 6 pixels, or $1.0 \times 1.0 \mu \mathrm{m}$ ) on a single neuronal soma was measured and analyzed. The pd1EGFP associated fluorescence was calculated by subtracting the fluorescence intensity in background (cell-free) area from the averaged intensity of three different ROIs on neuronal soma. ROIs were initially positioned by eye and corrected for the center of mass of each soma by an automated script in IPLab (Scanalytics). Before drug treatment, average intensities from 3 time frames were considered as an initial fluorescence level. Next, using time-lapse microscopy, images are collected and the fluorescence intensity of each frame was recorded. Fluorescence puncta in one neuron were pooled and averaged. Student t-test was used to analyze average intensity between groups.

\section{List of abbreviations}

(BDNF): Brain derived neurotrophic factor; (C.mycin): coumermycin; (GyrB): Gyrase B; (elF2): eukaryotic initiation factor-2; (LTP): long-term potentiation; (MODC): mouse ornithine decarboxylase; (NGF): nerve growth factor; (NT-3): neurotrophin-3; (NT-4/5): neurotrophin-4/5; (PI3K): phosphatidylinositol 3kinase; (PKR): double strand RNA dependent protein kinase; (SSCS): spontaneous synaptic currents.

\section{Acknowledgements}

We thank Drs. Guhan Nagappan and Newton Woo for helpful discussions and critical comments on the manuscript. We also thank Dr. Thomas E. Dever (NICHD, NIH) for the GyrB-PKR constructs and Regeneron Pharmaceuticals for recombinant NT-3.

\section{Author details}

${ }^{1}$ Genes, Cognition and Psychosis Program (GCAP), National Institute of Mental Health, NIH, Bethesda, MD 20892, U.S.A. ${ }^{2}$ Program in Neuroscience and Behavioural Disorders, DUKE-NUS Graduate Medical School, 169857 Singapore. ${ }^{3}$ GlaxoSmithKline, R\&D China, Pudong, Shanghai, 201203, China. ${ }^{4}$ School of Life Sciences, Tsinghua University, Beijing 100084, China. ${ }^{5}$ Protein Science Laboratory of the Ministry of Education, Tsinghua University, Beijing 100084, China.

\section{Authors' contributions}

HSJ, YW, and FY performed experiments. HSJ and YJ designed experiments and analyzed data. WW participated in the design and coordination of the study. HSJ, YJ, and BL wrote the manuscript. All authors read and approved the final version of a manuscript.

\section{Competing interests}

The authors declare that they have no competing interests.

Received: 24 December 2010 Accepted: 7 January 2011

Published: 7 January 2011

\section{References}

1. Goelet P, Castellucci VF, Schacher S, Kandel ER: The long and the short of long-term memory-a molecular framework. Nature 1986, 322:419-422.

2. McAllister AK, Katz LC, Lo DC: Neurotrophins and synaptic plasticity. Annu Rev Neurosci 1999, 22:295-318.

3. Poo MM: Neurotrophins as synaptic modulators. Nat Rev Neurosci 2001, 2:24-32.

4. Lu B: BDNF and activity-dependent synaptic modulation. Learning and Memory 2003, 10:86-98.

5. Lu B: Acute and long-term regulation of synapses by neurotrophins. Progress Brain Res 2004, 146:137-150.

6. Je HS, Zhou J, Yang F, Lu B: Distinct mechanisms for neurotrophin-3induced acute and long-term synaptic potentiation. J Neurosci 2005, 25:11719-11729.

7. Davies H, Squire L: Protein synthesis and memory: a review. Psychol Bull 1984, 96:518-599.

8. Je H-S, Yang F, Zhou J, Lu B: Neurotrophin 3 induces structural and functional modification of synapses through distinct molecular mechanisms. J Cell Biol 2006, 175:1029-1042.

9. Kundel M, Jones KJ, Shin CY, Wells DG: Cytoplasmic polyadenylation element-binding protein regulates neurotrophin-3-dependent betacatenin mRNA translation in developing hippocampal neurons. $J$ Neurosci 2009, 29:13630-13639.

10. Je HS, Lu Y, Yang F, Nagappan G, Zhou J, Jiang Z, Nakazawa K, Lu B: Chemically inducible inactivation of protein synthesis in genetically targeted neurons. J Neurosci 2009, 29:6761-6766.

11. Klann E, Dever TE: Biochemical mechanisms for translational regulation in synaptic plasticity. Nat Rev Neurosci 2004, 5:931-942.

12. Wu S, Kaufman RJ: A model for the double-stranded RNA (dsRNA)dependent dimerization and activation of the dsRNA-activated protein kinase PKR. J Biol Chem 1997, 272:1291-1296.

13. Ung TL, Cao C, LU J, Ozato K, Dever TE: Heterologous dimerization domains functionally substitute for the double-stranded RNA binding domains of the kinase PKR. Embo J 2001, 20:3728-3737.

14. Sidhu JS, Omiecinski CJ: Protein synthesis inhibitors exhibit a nonspecific effect on phenobarbital-inducible cytochome P450 gene expression in primary rat hepatocytes. J Biol Chem 1998, 273:4769-4775.

15. Xiong W, Kojic LZ, Zhang L, Prasad SS, Douglas R, Wang Y, Cynader MS: Anisomycin activates p38 MAP kinase to induce LTD in mouse primary visual cortex. Brain Res 2006, 1085:68-76.

16. Mohi MG, Arai K, Watanabe S: Activation and functional analysis of Janus kinase 2 in BA/F3 cells using the coumermycin/gyrase B system. Mol Biol Cell 1998, 9:3299-3308

17. Wang T, Xie KW, Lu B: Neurotrophins promote maturation of developing neuromuscular synapses. J Neurosci 1995, 15:4796-4805.

18. Li X, Zhao X, Fang Y, Jiang X, Duong T, Fan C, Huang CC, Kain SR: Generation of destabilized green fluorescent protein as a transcription reporter. J Biol Chem 1998, 273:34970-34975.

19. Mateus C, Avery SV: Destabilized green fluorescent protein for monitoring dynamic changes in yeast gene expression with flow cytometry. Yeast 2000, 16:1313-1323.

20. Lohof AM, Ip NY, Poo MM: Potentiation of developing neuromuscular synapses by the neurotrophins NT-3 and BDNF. Nature 1993, 363:350-353. 
21. Yang F, He X, Feng L, Mizuno K, Liu XW, Russell J, Xiong WC, Lu B: PI-3 kinase and IP3 are both necessary and sufficient to mediate NT3induced synaptic potentiation. Nat Neurosci 2001, 4:19-28.

22. Jiang Z, Belforte JE, Lu Y, Yabe Y, Pickel J, Smith CB, Je HS, Lu B, Nakazawa K: elF2alpha Phosphorylation-dependent translation in CA1 pyramidal cells impairs hippocampal memory consolidation without affecting general translation. J Neurosci 2010, 30:2582-2594.

23. Chang S, Popov SV: Long-range signaling within growing neurites mediated by neurotrophin-3. Proc Natl Acad Sci USA 1999, 96:4095-4100.

24. Martin KC, Casadio A, Zhu H, E Y, Rose JC, Chen M, Bailey CH, Kandel ER: Synapse-specific, long-term facilitation of aplysia sensory to motor synapses: a function for local protein synthesis in memory storage. Cell 1997, 91:927-938.

25. Beaumont V, Zhong N, Fletcher R, Froemke RC, Zucker RS: Phosphorylation and local presynaptic protein synthesis in calcium- and calcineurindependent induction of crayfish long-term facilitation. Neuron 2001, 32:489-501.

26. Frey U, Krug M, Reymann KG, Matthies H: Anisomycin, an inhibitor of protein synthesis, blocks late phases of LTP phenomena in the hippocampal CA1 region in vitro. Brain Res 1988, 452:57-65.

27. Loetscher P, Pratt $G$, Rechsteiner $M$ : The $C$ terminus of mouse ornithine decarboxylase confers rapid degradation on dihydrofolate reductase. Support for the pest hypothesis. J Biol Chem 1991, 266:11213-11220.

doi:10.1186/1756-6606-4-1

Cite this article as: Je et al.: Presynaptic protein synthesis required for NT-3-induced long-term synaptic modulation. Molecular Brain 2011 4:1.

\section{Submit your next manuscript to BioMed Central and take full advantage of:}

- Convenient online submission

- Thorough peer review

- No space constraints or color figure charges

- Immediate publication on acceptance

- Inclusion in PubMed, CAS, Scopus and Google Scholar

- Research which is freely available for redistribution

Submit your manuscript at www.biomedcentral.com/submit 